\title{
Das Projekt PRIMA - Elektronische Erstellung und Aktualisierung von Medikationsplänen als gemeinsame Aufgabe von Ärzten und Apothekern
}

\section{The PRIMA Project - Electronically-Supported Physician- Pharmacist Cooperation to Generate and Update Medication Plans in Germany}

Autoren

Christiane Eickhoff', Uta Müller ${ }^{1}$, Ann Kathrin Strunz' ${ }^{1}$, Hanna M. Seidling², Anette Lampert², Miriam Felberg ${ }^{1}$, Sabine Breiholz', Dirk Klintworth', Martin Schulz ${ }^{1,3}$

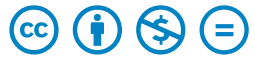

Institute

1 Geschäftsbereich Arzneimittel, ABDA Bundesvereinigung Deutscher Apothekerverbände e.V., Berlin

2 Klinische Pharmakologie und Pharmakoepidemiologie, Kooperationseinheit Klinische Pharmazie, Universitätsklinikum Heidelberg

3 Klinische Pharmazie und Biochemie, Institut für Pharmazie, Freie Universität Berlin

Schlüsselwörter

E-Health, Arzt-Apotheker-Zusammenarbeit, Arzneimitteltherapiesicherheit, elektronischer Medikationsplan

Key words

e-health, physician-pharmacist cooperation, medication safety, electronic medication plan

Bibliografie

DOI https://doi.org/10.1055/a-0859-5862

Dtsch Med Wochenschr 2019; 144: e114-e120

(c) Georg Thieme Verlag KG, Stuttgart · New York ISSN 0012-0472

Korrespondenzadresse

Dr. Christiane Eickhoff

Geschäftsbereich Arzneimittel

ABDA - Bundesvereinigung Deutscher

Apothekerverbände e. V., Unter den Linden 19 - 23,

10117 Berlin

armin@abda.de

\section{ZUSAMMENFASSUNG}

Einleitung Der Gesamtüberblick über die Medikation eines Patienten ist wesentliche Voraussetzung für die Arzneimitteltherapiesicherheit. Ein aktueller und vollständiger Medikationsplan (MP) ist hierfür ein geeignetes Instrument. Ziel war zu untersuchen, inwiefern die elektronische Bearbeitung des bundeseinheitlichen Medikationsplans gemeinsam durch Arzt und Apotheker in ihren Primärsystemen technisch machbar ist, die definierten Prozesse praktikabel sind und akzeptiert werden.

Methoden Ärzte und Apotheker testeten in einem mehrstufigen Verfahren die elektronische Umsetzung. Die Bearbeitung der MP erfolgte anhand definierter Prozesse und Verantwortlichkeiten; Machbarkeit und Akzeptanz wurden in schriftlichen Befragungen und einem Workshop evaluiert.

Ergebnisse Erstmalig in Deutschland wurden die Voraussetzungen zur elektronischen Erstellung, zum Austausch und zur Aktualisierung von MP in einem Arzt- und vier Apothekenverwaltungssystemen geschaffen. Gelöste Probleme waren technische und AMTS-relevante Fehler, abweichende Darstellungen, mangelnde Benutzerfreundlichkeit und begrenzte Speicherkapazität des Barcodes. Elf Arzt-Apotheker-Teams betreuten 196 Patienten. Insgesamt waren $60 \%$ mit den Prozessen und $80 \%$ der Ärzte sowie $63 \%$ der Apotheker mit den Verantwortlichkeiten zufrieden. Ärzte bewerteten die Erfassung der Gesamtmedikation durch Apotheker positiv. Die Kooperation verbesserte den fachlichen Austausch. Dies führt bei $70 \%$ der Ärzte bei der Medikation und bei $88 \%$ der Apotheker zum Gesundheitszustand der Patienten zu einem Wissenszuwachs. Alle Ärzte und Apotheker sahen eine strukturierte Zusammenarbeit als wichtig (25\%) oder sehr wichtig (75\%) für die Qualität der MP an.

Diskussion Der MP wurde erstmalig in Primärsysteme implementiert. Prozesse und Verantwortlichkeiten fanden bei Ärzten und Apothekern Akzeptanz. Dies sind wichtige Voraussetzungen, um den MP flächendeckend und nachhaltig zu implementieren.

\section{ABSTRACT}

Background A complete overview on the patient's medication is one precondition for medication safety. For this, a complete and current medication plan (MP) is an appropriate instrument. We aimed to develop and implement software to evaluate and exchange medication plans in local software systems of general practitioners (GPs) and community phar- 
macies (CPs). Furthermore, it was the aim to evaluate feasibility and acceptance of the defined processes.

Methods CPs and GPs were involved to pilot the software in several steps. Additionally, they generated and updated MP according to pre-defined processes and responsibilities. Feasibility and acceptance were evaluated in a survey and a workshop. Results For the first time in Germany, the technical requirements were established to generate and exchange MP electronically. Four software systems of CPs and one software system of GPs were involved. Solved Problems were technical errors, errors relevant for medication safety, differences in display of the medication data, and limited capacity of the barcode on the MP printout. Eleven GP and CP teams recruited 196 patients. $60 \%$ were satisfied with the defined proces- ses. $80 \%$ of the GPs and $63 \%$ of CPs agreed with the defined responsibilities. GPs considered the initial compilation on patient's medication in the CP as useful. The professional exchange between GPs and CPs improved: $70 \%$ of GPs referred to increased knowledge on medication and $88 \%$ of CPs received more information on patients' health conditions. The structured collaboration between GPs and CPs was considered to be important ( $25 \%$ ) or very important (75\%) for the quality of medication plans.

Discussion An electronic MP was successfully implemented for the first time in local software systems. Processes and responsibilities were accepted by both professions. These are important prerequisites for sustainably implementing the MP in daily practice.

\section{Einleitung}

Die Therapie mit Arzneimitteln ist ein komplexer Prozess, bei dem aufgrund einer Vielzahl von Beteiligten der Informationsaustausch von zentraler Bedeutung für die Patientensicherheit ist [1, 2]. Der fehlende Überblick über die Gesamtmedikation des Patienten ist eine häufige Ursache von Medikationsfehlern [3]; kritische Situationen wie Interaktionen oder unerwünschte Arzneimittelwirkungen werden dadurch häufig nicht rechtzeitig erkannt. Eine Lösung hierfür ist die interprofessionelle Nutzung eines Medikationsplans (MP) [4]. In Deutschland wurde im Rahmen der Aktionspläne zur Verbesserung der Arzneimitteltherapiesicherheit (AMTS) des Bundesministeriums für Gesundheit (BMG) ein Standard für einen solchen MP entwickelt, der sogenannte bundeseinheitliche Medikationsplan (BMP) [5]. Bei Einführung des BMP wurde aber nicht festgelegt, durch welche Prozesse die Erstellung und Aktualisierung begleitet werden sollte. Das BMG förderte daher drei Projekte zur „Erprobung der Machbarkeit und Akzeptanz des BMP“. Dies waren die Projekte „MetropolMediplan 2016“ in Nürnberg/Erlangen/Fürth, die Modellregion Erfurt und das Projekt „PRIMA“ (Primärsystem-Integration des Medikationsplans mit Akzeptanzuntersuchung) in Sachsen und Thüringen. Die gesammelten Erkenntnisse und abgeleiteten Empfehlungen zeigen einheitlich, dass Apotheker in die Erstellung und Fortschreibung von MP eingebunden werden müssen, dass Prozesse und Zuständigkeiten klar definiert werden müssen und dass der BMP in die Primärsoftware von Arztpraxen, Apotheken und Krankenhäusern integriert werden muss [6].

PRIMA untersuchte als einziges dieser Projekte, wie eine solche Integration in die Software von Arztpraxen und Apotheken umgesetzt werden kann, wie zugehörige Prozesse bewertet und deren Machbarkeit und Akzeptanz von Ärzten und Apothekern eingestuft werden [7]. Partner in dem Projekt waren die ABDA Bundesvereinigung Deutscher Apothekerverbände e. V., die Kassenärztliche Bundesvereinigung (KBV), die AOK PLUS sowie die Kassenärztlichen Vereinigungen und Landesapothekerverbände in Sachsen und Thüringen. In PRIMA konnte auf die im Modellvorhaben „Arzneimittelinitiative Sachsen-Thüringen (ARMIN)“ [8] erarbeiteten Standards für ein interprofessionelles Medikations- management (MM) zurückgegriffen werden. Dazu zählten die technische Infrastruktur zum sicheren Datenaustausch zwischen Arztpraxis und Apotheke sowie die Prozesse zur Arzt-ApothekerZusammenarbeit, einschließlich der Erstellung und Aktualisierung von MP [9].

Ziel des Projektes PRIMA war die Pilotierung der Betreuung der ersten ARMIN-Patienten unter Einbeziehung der technischen Infrastruktur. Dabei wurde untersucht, inwiefern die elektronische Bearbeitung des BMP technisch machbar ist, die definierten Prozesse praktikabel sind und von Ärzten und Apothekern akzeptiert werden.

\section{Material und Methoden}

Das Projekt wurde von Oktober 2014 bis März 2017 in je 12 an ARMIN teilnehmenden Apotheken und Arztpraxen in Sachsen und Thüringen durchgeführt. Alle hatten an einer gemeinsamen Qualifikationsveranstaltung teilgenommen.

Nachdem die technischen Voraussetzungen zum elektronischen Austausch von MP geschaffen waren, sollte jedes ArztApotheker-Team 10 Patienten rekrutieren. Die Teilnahme stand nur Versicherten der AOK PLUS offen.

Die Erstellung und Aktualisierung der MP erfolgte in einem strukturierten Prozess mit definierten Verantwortlichkeiten für beide Heilberufler [8, 9]. Die pharmazeutische Intervention zur Erfassung der Gesamtmedikation mit pharmazeutischer AMTSPrüfung erfolgte gemäß Leitlinie „Medikationsanalyse“ der Bundesapothekerkammer [10]. Die medizinische AMTS-Prüfung einschließlich Priorisierung der Medikation orientierte sich an der „Hausärztlichen Leitlinie Multimedikation“ der Deutschen Gesellschaft für Allgemeinmedizin und Familienmedizin [11].

Positive Ethikvoten der Landesärztekammern Sachsen und Thüringen lagen zum Projektbeginn vor.

\section{Machbarkeit der elektronischen Erstellung}

Die MP wurden in neu entwickelten Softwaremodulen erstellt und aktualisiert, die in die Praxisverwaltungssysteme (PVS) bzw. Apothekenverwaltungssysteme (AVS) integriert waren. Form und 


\begin{tabular}{|c|c|c|c|c|c|c|c|c|c|c|}
\hline \multicolumn{2}{|c|}{$\begin{array}{l}\text { Medikationsplan } \\
\text { Seite } 1 \text { von } 1\end{array}$} & \multicolumn{8}{|c|}{ 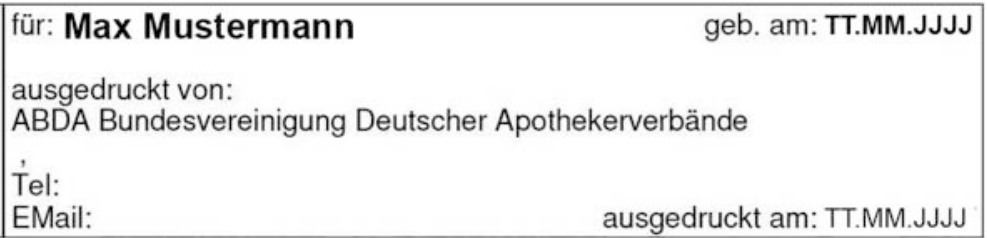 } & \\
\hline Wirkstoff & \multicolumn{2}{|c|}{ Handelsname } & Stärke & Form & $\begin{array}{l}\text { mor. } \\
\text { gens }\end{array}$ & & abends zur & Einheit & Hinweise & Grund \\
\hline $\begin{array}{l}\text { Insulin } \\
\text { normal (human) }\end{array}$ & \multicolumn{2}{|c|}{$\begin{array}{l}\text { Actrapid Penfill 100l.E./ml } \\
\text { Zylinderampullen }\end{array}$} & $100 \mathrm{IE} / \mathrm{ml}$ & Amp & 10 & 6 & $8 \quad 0$ & IE & vor den Mahlzeiten, nach Messergebnis & Diabetes mellitus \\
\hline Insulin glargin & \multicolumn{2}{|c|}{$\begin{array}{l}\text { Lantus } 100 \mathrm{E} / \mathrm{ml} \text { SoloStar } \\
\text { Fertigpen }\end{array}$} & $100 \mathrm{E} / \mathrm{ml}$ & Spritze & \multicolumn{3}{|c|}{ Siehe Hinweis } & IE & Abends 28-30 IE nach Messergebnis & Diabetes mellitus \\
\hline Metformin hydrochlorid & \multicolumn{2}{|c|}{$\begin{array}{l}\text { Metformin Lich } 1000 \mathrm{mg} \\
\text { Filmtabletten }\end{array}$} & $1000 \mathrm{mg}$ & Tabl & 1 & 0 & $\begin{array}{ll}1 & 0\end{array}$ & Stück & $\begin{array}{l}\text { zu oder unmittelbar nach den } \\
\text { Mahlzeiten }\end{array}$ & Diabetes mellitus \\
\hline Levothyroxin natrium & \multicolumn{2}{|c|}{ L THYROX HEXAL 100} & $0,1 \mathrm{mg}$ & Tabl & 0,5 & 0 & \begin{tabular}{l|l}
0 & 0 \\
\end{tabular} & Stück & 30 min vor dem Frühstück & $\begin{array}{l}\text { Schilddrūsenunterfunktio } \\
\text { n }\end{array}$ \\
\hline Torasemid & \multicolumn{2}{|c|}{$\begin{array}{l}\text { Torasemid AL 10mg } \\
\text { Tabletten }\end{array}$} & $10 \mathrm{mg}$ & Tabl & 1 & 1 & $0 \quad 0$ & tück & & Wassereinlagerung Beine \\
\hline $\begin{array}{l}\text { Ramipril } \\
\text { Hydrochlorothiazid }\end{array}$ & \multicolumn{2}{|c|}{$\begin{array}{l}\text { Ramipril comp. AbZ } \\
5 \mathrm{mg} / 25 \mathrm{mg} \text { Tabletten }\end{array}$} & $\begin{array}{r}5 \mathrm{mg} \\
25 \mathrm{mg} \\
\end{array}$ & Tabl & 1 & 0 & 0 & Stück & $\begin{array}{l}\text { ggf. bei weiter niedrigem Blutdruck früh } \\
\text { nur } 0,5\end{array}$ & Bluthochdruck \\
\hline Bisoprolol hemifumarat & \multicolumn{2}{|c|}{$\begin{array}{l}\text { Bisoprolol AbZ } 5 \mathrm{mg} \\
\text { Tabletten }\end{array}$} & $5 \mathrm{mg}$ & Tabl & 1 & 0 & \begin{tabular}{l|l}
0 & 0 \\
\end{tabular} & Stück & & Bluthochdruck \\
\hline
\end{tabular}

Bedarfsmedikation

\begin{tabular}{|l|l|r|l|l|l|l|l|l|l|l|l|}
\hline Diclofenac natrium & Diclo 50-1A Pharma & $50 \mathrm{mg}$ & Tabl & \multicolumn{3}{|l|}{ Bei Bedarf 1Tbl } & Stūck & nur im Bedarfsfall & Schmerzen \\
\hline Metamizol natrium & $\begin{array}{l}\text { NOVAMINSULFON } \\
\text { 500MG LICHT }\end{array}$ & $\begin{array}{r}500 \\
\mathrm{mg} / \mathrm{ml}\end{array}$ & Tropfen & 30 & 30 & 30 & 0 & Tropfen & nur im Bedarfsfall & Schmerzen \\
\hline
\end{tabular}

Inhalt der MP entsprachen dem von der Koordinierungsgruppe AMTS entwickelten BMP ( $\triangleright$ Abb. 1) [5].

Zusätzlich waren Kommentarfelder für eine weitergehende elektronische Arzt-Apotheker-Kommunikation enthalten. Als Datenformat wurde das für ARMIN entwickelte Medikationsplanaustauschformat verwendet. Der Austausch der MP zwischen den Praxen und Apotheken erfolgte über einen Server im sicheren Netz der Kassenärztlichen Vereinigungen (KV-SafeNet, der Begriff KV-SafeNet steht nicht mit der Firma „SafeNet, Inc., USA“ in firmenmäßiger oder vertraglicher Verbindung) [9].

Die ersten Tests wurden auf Ebene der Softwarehäuser zwischen AVS und PVS durchgeführt. Dazu wurden entsprechende Testszenarien entwickelt, die alle überprüfbaren Anforderungen enthielten. Im nächsten Schritt wurde der Datenaustausch an einem Standardfall zwischen Arztpraxen und Apotheken erprobt. Im dritten Schritt erfolgte erstmalig, unter engmaschigem Monitoring, der Austausch echter MP für je 2 Patienten je Arzt-Apotheker-Team. Abschließend sollten je Arzt-Apotheker-Team bis zu 10 Patienten in der Routine betreut werden.

\section{Erhebung von Praktikabilität und Akzeptanz der Medikationsplan-bezogenen Prozesse bei Ärzten und Apothekern}

Praktikabilität und Akzeptanz wurden mit Fragebögen und während eines Workshops erfasst.

\section{Fragebögen}

Die Fragebögen für Ärzte und Apotheker umfassten jeweils 11 Fragen mit 42 Items zur Motivation der Teilnahme, der Zufriedenheit mit der technischen Umsetzung, den Prozessen im MM, dem Projektnutzen und der Verwendung der MP durch die Patienten. Weiterhin wurden Daten zu Berufserfahrung und EDV-Kenntnissen erhoben. Die Fragebögen wurden hinsichtlich Verständlichkeit und Bearbeitungszeit pilotiert und den Teilnehmern im August 2016 postalisch zugeschickt. Eine Erinnerung erfolgte per E-Mail.

\section{Workshop}

Im September 2016 fand ein Workshop statt, während dessen Inhaber und Mitarbeiter der teilnehmenden Arztpraxen und Apotheken die Teilschritte der Erstellung und Pflege von MP bewerteten und diskutierten. Zudem erfolgte eine anonyme elektronische Abstimmung zu Aspekten des BMP, der kurz nach dem Workshop-Termin zum 01.10.2016 mit dem E-Health- 
Gesetz nach § 31 a SGB V eingeführt wurde (Abstimmungssystem: TurningPoint ${ }^{\circledR}$ by Turning Technologies, Version 5.4.1.2). Basierend auf ihren Erfahrungen in PRIMA sollten die Teilnehmer die Erstellung des BMP gemäß E-Health-Gesetz bewerten, da dort keine Vorgaben zu Prozessen gemacht wurden.

\section{Statistik}

Die Antworten der Fragebögen wurden mit IBM ${ }^{\circledR}$ SPSS $^{\circledR}$ Statistics for Windows, Version 21 (IBM Corp., Armonk, N.Y., USA) deskriptiv (Häufigkeiten, Mittelwerte, Standardabweichungen) ausgewertet.

\section{Ergebnisse}

In 12 Arztpraxen und Apotheken in Sachsen und Thüringen wurden die technischen Voraussetzungen geschaffen, um MP im PVS bzw. AVS zu bearbeiten und mit dem anderen Heilberufler auszutauschen. 11 Arzt-Apotheker-Teams schlossen 196 Patienten ein. Ein Team schied aufgrund eines Wechsels des Softwareanbieters der Apotheke aus.

\section{Machbarkeit der elektronischen Erstellung und des Austausches von Medikationsplänen}

Die Umsetzung erfolgte in einer PVS-Produktlinie und 5 Produktlinien von 4 AVS. Entsprechend wurden alle Tests für die 5 möglichen PVS-AVS-Kombinationen durchgeführt.

Die identifizierten Probleme sind 5 Kategorien zuzuordnen:

(1) Technische, den Ablauf verzögernde oder verhindernde Probleme

Ursache waren Interpretationsprobleme von Daten zwischen AVS, PVS und Medikationsplanserver durch Abweichungen vom zulässigen Zeichensatz oder Datenformat. Kommunikationsprobleme zwischen Softwaremodulen und dem Server traten durch serverseitige Prüfung auf Validität oder bei der Datenverarbeitung auf Basis unterschiedlicher Arzneimitteldatenbanken auf, da unterschiedliche Softwarehäuser mit unterschiedlichen Arzneimitteldatenbanken (MMI, ifap, ABDATA) arbeiten.

\section{(2) AMTS-relevante Fehler}

Programmierfehler führten zu fehlenden Zeilen in MP, fehlenden Handelsnamen sowie fehlenden Wirkstoffmengen oder Maßeinheiten.

\section{(3) Unterschiedliche Darstellungen identischer Medika- tionsplaneinträge}

Inhaltlich identische Einträge wurden unterschiedlich dargestellt. Dies war entweder die Folge unterschiedlicher Umsetzungen verschiedener Softwareanbieter oder resultierte aus Unterschieden in den Arzneimitteldatenbanken.

Beispiele für festgestellte Abweichungen:

- variierende Wirkstoff- oder Stärkeangaben durch unterschiedliche Wirkstoffbezüge, z. B.: Bisoprolol 4,24 mg versus Bisoprolol $5 \mathrm{mg}$ versus Bisoprolol hemifumarat $5 \mathrm{mg}$
- Bezug auf unterschiedliche Einheiten bei der Stärkeangabe, z. B.: Levothyroxin natrium 0,1 mg versus Levothyroxin $100 \mu \mathrm{g}$

- scheinbar unterschiedliche Stärken durch unterschiedliche Volumenbezüge, z. B.: Insulin glargin 100 I.E./ml versus Insulin glargin 300 I.E. (Bezug auf gesamte Zylinderampulle mit $3 \mathrm{ml}$ Inhalt)

Im verwendeten Standardfall mit 9 Arzneimitteln ergaben sich durch die Verwendung zweier unterschiedlicher Arzneimitteldatenbanken abweichende Darstellungen bei 6 Wirkstoffen, einem Handelsnamen und 3 Stärkenangaben ( $\triangleright$ Abb. 2).

\section{(4) Benutzerfreundlichkeit der Software}

Die Benutzerfreundlichkeit war mitentscheidend für die Akzeptanz. Anwender bemängelten beispielsweise, dass gleiche MP-Zeilen aufgrund nicht standardisierter Arzneimitteldaten nicht als gleich erkannt wurden, dass Änderungen nicht hervorgehoben wurden und dass eine Gesamtübersicht aller MP mit Filtermöglichkeiten nach Bearbeitungsstatus sowie eine automatisierte Identifikation geeigneter Projektteilnehmer fehlen würde. Weiterhin bestand der Wunsch nach standardisierten Textbausteinen.

\section{(5) Begrenzte Speicherkapazität des Barcodes im Ausdruck} des BMP

Obwohl MP-Seiten optisch nicht vollständig befüllt waren, ergaben sich Seitenumbrüche, da die Speicherkapazität des Barcodes ausgeschöpft war. Dies führte zu Irritationen.

Die Behebung technischer und AMTS-relevanter Probleme war notwendig, um Richtigkeit und Vollständigkeit der MP zu gewährleisten. Entsprechende technische Anpassungen und Klarstellungen wurden vorgenommen. Die Umsetzung dauerte für die unterschiedlichen Softwareprodukte ca. 1 - 2 Monate.

Abweichungen aufgrund der unterschiedlichen Arzneimitteldatenbanken wurden an die nach §31a SGB V zur Ausgestaltung des BMP beauftragten Spitzenorganisationen (KBV, Bundesärztekammer, Deutscher Apothekerverband e. V.) mit Bitte um Prüfung und Bearbeitung kommuniziert. Soweit eine Lösung ohne vollständige Standardisierung der Daten möglich war, wurde die BMP-Spezifikation angepasst [5].

Anregungen zur Verbesserung der Benutzerfreundlichkeit wurden an die Softwarehersteller übermittelt.

Im Hinblick auf die begrenzte Speicherkapazität des Barcodes wurden die Teilnehmer angehalten, dies durch kurze Freitexte zu vermeiden oder ihre Patienten darüber aufzuklären.

\section{Praktikabilität und Akzeptanz der Prozesse durch Ärzte und Apotheker}

Acht Apotheker und 10 Ärzte nahmen an der schriftlichen Befragung teil (Rücklaufquoten 71 bzw. 91 \%). Alle Ärzte und 7/8 Apotheker verfügten über mindestens 5 Jahre Berufserfahrung. Ihre EDV-Kenntnisse bewerteten die Ärzte mit der Schulnote 2,3 $( \pm 0,7)$, die Apotheker mit 2,6 $( \pm 1,1)$.

Am Workshop nahmen 35 Personen aus je 10 Apotheken und Arztpraxen teil (14 Apotheker, 15 Ärzte und 6 Praxismitarbeiter). 

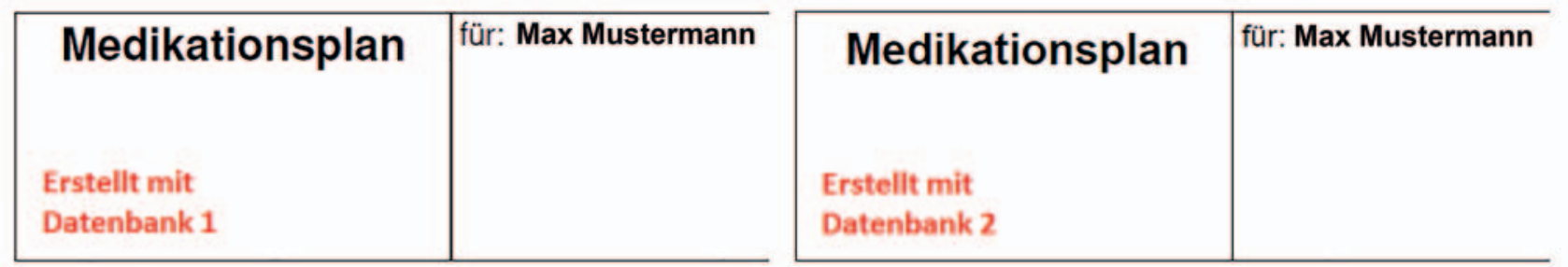

\begin{tabular}{|l|l|r|l|}
\hline Wirkstoff & Handelsname & Stärke & Form \\
\hline $\begin{array}{l}\text { Insulin } \\
\text { normal (human) }\end{array}$ & $\begin{array}{l}\text { Actrapid Penfill 100l.E/ml } \\
\text { Zylinderampullen }\end{array}$ & $100 \mathrm{IE} / \mathrm{ml}$ & Amp \\
\hline Insulin glargin & $\begin{array}{l}\text { Lantus 100 E/ml SoloStar } \\
\text { Fertigpen }\end{array}$ & $100 \mathrm{E} / \mathrm{ml}$ & Spritze \\
\hline Mettormin hydrochlorid & $\begin{array}{l}\text { Mettormin Lich 1000mg } \\
\text { Filmtabletten }\end{array}$ & $1000 \mathrm{mg}$ & Tabl \\
\hline Levothyroxin natrium & L THYROX HEXAL 100 & $0,1 \mathrm{mg}$ & Tabl \\
\hline Torasemid & $\begin{array}{l}\text { Torasemid AL 10mg } \\
\text { Tabletten }\end{array}$ & $10 \mathrm{mg}$ & Tabl \\
\hline $\begin{array}{l}\text { Ramipril } \\
\text { Hydrochlorothiazid }\end{array}$ & $\begin{array}{l}\text { Ramipril comp. AbZ } \\
\text { 5mg/25mg Tabletten }\end{array}$ & $\begin{array}{r}5 \mathrm{mg} \\
25 \mathrm{mg}\end{array}$ & Tabl \\
\hline Bisoprolol hemifumarat & $\begin{array}{l}\text { Bisoprolol AbZ 5 mg } \\
\text { Tabletten }\end{array}$ & $5 \mathrm{mg}$ & Tabl \\
\hline
\end{tabular}

Bedarfsmedikation

\begin{tabular}{|l|l|r|l|}
\hline Diclofenac natrium & Diclo 50-1A Pharma & $50 \mathrm{mg}$ & Tabl \\
\hline Metamizol natrium & $\begin{array}{l}\text { NOVAMINSULFON } \\
500 M G \text { LICHT }\end{array}$ & $\begin{array}{r}500 \\
\mathrm{mg} / \mathrm{ml}\end{array}$ & Tropfen \\
\hline
\end{tabular}

\begin{tabular}{|c|c|c|c|}
\hline Wirkstoff & Handelsname & Stärke & Form \\
\hline Insulin, normal & $\begin{array}{l}\text { ACTRAPID Penfill } 100 \\
\text { I.E./ml Ini.-Lsq i.Patrone }\end{array}$ & 300I.E. & Amp \\
\hline Insulin glargin & $\begin{array}{l}\text { LANTUS } 100 \mathrm{E} / \mathrm{ml} \\
\text { SoloStar Fertigpen }\end{array}$ & $300 \mathrm{E}$. & Spritze \\
\hline Metformin & $\begin{array}{l}\text { METFORMIN Lich } 1.000 \\
\text { mg Filmtabletten }\end{array}$ & $1000 \mathrm{mg}$ & Tabl \\
\hline Levothyroxin & $\begin{array}{l}\text { L-THYROX HEXAL } 100 \\
\text { Tabletten }\end{array}$ & $0,1 \mathrm{mg}$ & Tabl \\
\hline Torasemid & $\begin{array}{l}\text { TORASEMID AL } 10 \text { mg } \\
\text { Tabletten }\end{array}$ & $10 \mathrm{mg}$ & Tabl \\
\hline $\begin{array}{l}\text { Ramipril + } \\
\text { Hydrochlorothiazid }\end{array}$ & $\begin{array}{l}\text { RAMIPRIL comp. AbZ } 5 \\
\text { mg/25 mg Tabletten }\end{array}$ & $\begin{array}{l}5 \mathrm{mg}+ \\
25 \mathrm{mg}\end{array}$ & Tabl \\
\hline Bisoprolol & $\begin{array}{l}\text { BISOPROLOL AbZ } 5 \mathrm{mg} \\
\text { Tabletten }\end{array}$ & $5 \mathrm{mg}$ & Tabl \\
\hline \multicolumn{4}{|c|}{ Bedarfsmedikation } \\
\hline Diclofenac & $\begin{array}{l}\text { DICLO } 501 \mathrm{~A} \text { Pharma } \\
\text { magensaftresistente... }\end{array}$ & $50 \mathrm{mg}$ & Tabl \\
\hline Metamizol & $\begin{array}{l}\text { NOVAMINSULFON } 500 \\
\text { mg Lichtenst. Tropfen... }\end{array}$ & $500 \mathrm{mg}$ & Tropfen \\
\hline
\end{tabular}

- Abb.2 Exemplarische Gegenüberstellung von Medikationsplan-Ausschnitten des Standardfalls, erstellt auf Basis zweier unterschiedlicher Arzneimitteldatenbanken.

\section{Praktikabilität der Erstellung, Nutzung und Aktuali- sierung des Medikationsplans}

Etwa 2/3 der Teilnehmer der Befragung waren mit den Prozessen insgesamt zufrieden (Ärzte: 6/10, Apotheker: 5/8). 12/13 Ärzten und Praxismitarbeitern sowie alle Apotheker bewerteten die pharmazeutische Intervention zur Erfassung der Gesamtmedikation mit pharmazeutischer AMTS-Prüfung positiv [10]. Die ärztliche Intervention mit medizinischer AMTS-Prüfung und Priorisierung der Medikation [11] wurde von vielen Ärzten als herausfordernd bewertet. Weitergehende Software-Unterstützungen, beispielsweise durch Einbettung von Informationen zur Bewertung der Medikation (z. B. PRISCUS-Liste [12]), wurden gewünscht. Kritisiert wurden technische Probleme, wie z. B. eine unzureichende Server-Erreichbarkeit sowie der hohe Zeitaufwand bei der Erstellung der MP (Ärzte: 7/10, Apotheker: 5/8).

Positiv bewertet wurde von 8/10 Ärzten und 7/8 Apothekern die Verständlichkeit der MP für die Patienten.

Die Mehrheit der Ärzte und Apotheker gab an, dass Patienten ihren ausgedruckten MP in der Praxis/Apotheke häufig weder mitbringen noch unaufgefordert vorzeigen würden. Im Rahmen des Projekts konnten die Ärzte und Apotheker jedoch jederzeit elektronisch auf den aktuellen Medikationsplan zugreifen.

\section{Arzt-Apotheker-Kooperation}

Der Informationsaustausch zwischen den Heilberuflern erfolgte primär telefonisch (nachrangig: persönliche Treffen, Fax, E-Mail) und bezog sich zum Zeitpunkt des Workshops primär auf organi- satorische und technische Fragen. $80 \%$ der Teilnehmer bewerteten die Kommunikation positiv; negative Meinungen waren überwiegend auf technische Probleme zurückzuführen. Für den Routinebetrieb wurden die elektronischen Kommentarfelder als unverzichtbar erachtet. Aus Sicht beider Berufsgruppen haben sich durch das Projekt der fachliche Austausch und die Zusammenarbeit verbessert. 7/10 Ärzten erhielten außerdem mehr Informationen zur Selbst- und Facharztmedikation und 7/8 Apothekern zum Gesundheitszustand und den Erkrankungen der Patienten. 7/10 Ärzten gingen mittelfristig von einer Zeitersparnis aus.

Als häufigster Grund zur Projektteilnahme wurde angegeben, das MM als geeignete Maßnahme zur Verbesserung der AMTS zu sehen (Ärzte: 9/10, Apotheker: 8/8). 7/10 Ärzte gaben zudem die erwartete Zeitersparnis in der Patientenbetreuung und 7/8 Apotheker die Etablierung des MM als neues Aufgabengebiet als Motivation an.

\section{Vergleich der BMP-Erstellung in PRIMA und nach E-Health-Gesetz}

28 Personen nahmen an der elektronischen Umfrage im Rahmen des Workshops teil (10 Ärzte, 12 Apotheker, 6 Praxismitarbeiter).

Sowohl der BMP nach E-Health-Gesetz als auch der MP in PRIMA wurden als Instrument zur Erhöhung der AMTS eingestuft, der MP in PRIMA jedoch aufgrund der strukturierten Zusammenarbeit in größerem Ausmaß. Dies wurde von allen Teilnehmern als wichtig $(25 \% ; n=7)$ oder sehr wichtig $(75 \% ; n=21)$ für die Quali- 
tät der MP bewertet. Auch schätzen 79\% der Befragten ( $n=22$ ) den MP in PRIMA deshalb eher als vollständig ein, als den BMP nach E-Health-Gesetz.

Für die Mehrheit der Teilnehmer lohnt sich der höhere Aufwand zur Erstellung von MP im MM im Hinblick auf die Verbesserung der AMTS.

\section{Diskussion}

In PRIMA wurde erstmalig in Deutschland ein elektronischer MP nach einem standardisierten Prozess in den AVS und PVS erstellt und fortlaufend aktualisiert. Dafür wurde der BMP in bestehende Softwaresysteme integriert sowie eine Infrastruktur zum elektronischen Datenaustausch zwischen Arztpraxen und Apotheken aufgebaut. Vor allem die elektronische Unterstützung wurde sowohl von den beteiligten Ärzten und Apothekern als auch von den beiden anderen BMG-geförderten Modellprojekten als Voraussetzung für die Bearbeitung von MP im Versorgungsalltag bewertet [6]. So beschrieb das Projektteam „Modellregion Erfurt“ bei fehlender Software-Integration einen hohen Zeitaufwand für die Erstellung und Fortschreibung von MP auf ärztlicher Seite und vermutete, dass sich durch eine Integration in die Primärsysteme die Akzeptanz steigern ließe [6]. Das Projekt MetropolMediplan 2016 kam zu dem Schluss, dass die praktische Umsetzung des BMP hinter den Erwartungen zurückbliebe, da dieser bisher weder technisch noch prozessual in bestehende Systeme integriert sei. Dies sei aber Voraussetzung für eine hohe Nutzerakzeptanz [6].

Durch eine strukturierte Arzt-Apotheker-Zusammenarbeit lässt sich der Erfolg einer Arzneimitteltherapie verbessern [13, 14]. Ein gutes Vertrauensverhältnis, eine enge Kommunikation und ein klares Rollenverständnis sind dafür wichtige Voraussetzungen [15]. Auch die an PRIMA teilnehmenden Ärzte und Apotheker begrüßten die Festlegung der Verantwortlichkeiten und Abläufe und sahen eine Verbesserung ihrer Zusammenarbeit. Sie schätzten die Erstellung und regelmäßige Aktualisierung der MP überwiegend als praktikabel ein. Ein wesentlicher Faktor für Unzufriedenheit stellte vermutlich der hohe Zeitaufwand dar, der in diesem Projekt zwar nicht erhoben wurde, aber für eine vergleichbare Intervention im Median mit 90 min angegeben wird [16].

Die teilnehmenden Ärzte stellten bereits im Vorfeld des Projektes MP aus, sahen jedoch in der Erfassung der Gesamtmedikation durch den Apotheker einen Informationsgewinn, insbesondere zur Selbst- und Facharztmedikation. Beide Berufsgruppen sahen einen persönlichen Nutzen im fachlichen Austausch und für die Versorgungsqualität. Trotz des hohen Zeitaufwands und der technischen Herausforderungen bewerteten die Leistungserbringer ein interdisziplinäres MM als geeignete Maßnahme zur Verbesserung der AMTS und sprachen sich für eine Fortführung und Weiterentwicklung des Konzeptes aus.

Gleichzeitig zeigte PRIMA aber auch, dass die Entwicklung neuer technischer Strukturen einen erheblichen Aufwand und eine enge Abstimmung aller Beteiligten erfordert. Nur einfache und benutzerfreundliche Softwarelösungen finden Akzeptanz. Die in PRIMA als Kommunikationstool zwischen Leistungserbringern integrierten Kommentarfelder im MP sind ein Beispiel dafür.
Auf Basis der Ergebnisse aus PRIMA wurden die technischen Strukturen sowie die interdisziplinäre Erstellung und Pflege der MP im Rahmen von ARMIN in Sachsen und Thüringen seit Juli 2016 breiter ausgerollt. Das Modellvorhaben wird mindestens bis März 2022 fortgeführt [8, 9]. Ab 2019 wird in einer externen, wissenschaftlichen Evaluation untersucht, inwieweit der Ansatz einen Nutzen hinsichtlich therapierelevanter und ökonomischer Endpunkte hat.

\section{KERNAUSSAGEN}

- Voraussetzung für die Etablierung des bundeseinheitlichen Medikationsplans nach §31a SGB V in der Praxis ist die Möglichkeit zur elektronischen Erstellung und Aktualisierung in der Primärsoftware und der Austausch der Medikationsplandaten zwischen Ärzten und Apothekern.

- Prozesse und Verantwortlichkeiten von Arzt und Apotheker bei der Erstellung und Aktualisierung von Medikationsplänen müssen eindeutig geregelt werden.

- Die in ARMIN vereinbarten Verantwortlichkeiten von Arzt und Apotheker wurden als sinnvoll und praktikabel bewertet.

- Qualitätsanforderungen an Medikationspläne sind unter anderem: Vollständigkeit, Aktualität sowie die Prüfung der Medikation auf Risiken für die Arzneimitteltherapiesicherheit.

- Ärzte bewerteten die Erfassung und pharmazeutische Bewertung der Gesamtmedikation inkl. Selbstmedikation in der Apotheke positiv.

\section{Finanzielle Unterstützung}

Diese Arbeit wurde als Teil des Projektes PRIMA vom BMG gefördert.

Interessenkonflikt

Die Autoren geben an, dass kein Interessenkonflikt besteht.

\section{Danksagung}

Die Autoren danken allen Arztpraxen, Apotheken und Projektpartnern für ihr großes Engagement sowie dem BMG für die finanzielle Förderung.

\section{Literatur}

[1] Pirmohamed $M$, James S, Meakin S et al. Adverse drug reactions as cause of admission to hospital: prospective analysis of 18820 patients. BM] 2004; 329: 15-19. doi:10.1136/bmj.329.7456.15

[2] Stausberg J. International prevalence of adverse drug events in hospitals. An analysis of routine data from England, Germany, and the USA. BMC Health Serv Res 2014; 14: 125. doi:10.1186/1472-6963-14-125

[3] Owen MC, Chang NM, Chong DH et al. Evaluation of medication list completeness, safety, and annotations. AMIA Annu Symp Proc 2011; 2011: $1055-1061$

[4] Kuske S, Lessing C, Lux R et al. Patientensicherheitsindikatoren zur Arzneimitteltherapiesicherheit (AMTS-PSI): Internationaler Status, Übertragbarkeit und Validierung*. Gesundheitswesen 2012; 74: 79-86. doi:10.1055/s-0030-1269838 
[5] BÄK, DAV, KBV. Spezifikation für einen bundeseinheitlichen Medikationsplan (BMP), Anlage 3. Im Internet: http://www.kbv.de/media/sp/ Medikationsplan_Anlage3.pdf; Stand: 26.03.2019

[6] Dormann H, Maas R, Eickhoff C et al. Der bundeseinheitliche Medikationsplan in der Praxis. Die Pilotprojekte MetropolMediplan 2016, Modellregion Erfurt und PRIMA. Bundesgesundheitsblatt 2018; 61: 1093- 1102

[7] Schulz M, Eickhoff C, Müller U. Erprobung eines Medikationsplans in der Praxis hinsichtlich der Akzeptanz und Praktikabilität. Pilotuntersuchung: Lesbarkeits- und Verständlichkeitstestung. Hauptuntersuchung PRIMA Primärsystem-Integration des Medikationsplans mit Akzeptanzuntersuchung [Abschlussbericht]. Im Internet: https://www.bundesgesund heitsministerium.de/fileadmin/Dateien/5_Publikationen/Gesundheit/ Berichte/PRIMA_Abschlussbericht.pdf; Stand: 01.10.2018

[8] ARMIN Arzneimittelinitiative Sachsen-Thüringen. Im Internet: http://www.arzneimittelinitiative.de; Stand: 26.03.2019

[9] Müller U, Schulz M, Mätzler M. Elektronisch unterstützte Kooperation ambulant tätiger Ärzte und Apotheker zur Verbesserung der Arzneimitteltherapiesicherheit. Die Arzneimittelinitiative Sachsen-Thüringen (ARMIN). Bundesgesundheitsblatt 2018; 61: 1119-1128. doi:10.1007/ s00103-018-2780-5

[10] Bundesapothekerkammer. Leitlinie der Bundesapothekerkammer zur Qualitätssicherung. Medikationsanalyse. Berlin, 2014

[11] Muth C, Beyer M, Popert U. Hausärztliche Leitlinie: Multimedikation. Empfehlungen zum Umgang mit Multimedikation bei Erwachsenen und geriatrischen Patienten (2013). Im Internet: http://www.pmvfor schungsgruppe.de/pdf/03_publikationen/multimedikation_II.pdf; Stand: 26.03 .2019

[12] Holt S, Schmiedl S, Thürmann PA. Potentially inappropriate medications in the elderly: the PRISCUS list. Dtsch Arztebl Int 2010; 107: 543 - 551. doi:10.3238/arztebl.2010.0543

[13] Hirsch JD, Steers N, Adler DS et al. Primary care-based, pharmacistphysician collaborative medication-therapy management of hypertension. A randomized, pragmatic trial. Clin Ther 2014; 36: 1244-1254. doi:10.1016/j.clinthera.2014.06.030

[14] Tobari H, Arimoto T, Shimojo N et al. Physician-pharmacist cooperation program for blood pressure control in patients with hypertension. A randomized-controlled trial. Am J Hypertens 2010; 23: 1144-1152. doi:10.1038/ajh.2010.127

[15] Weißenborn M, Schulz M, Kraft M et al. Potentielle Erfolgsindikatoren für die Durchführung von Projekten zur Arzt-Apotheker-Zusammenarbeit eine systematische Übersicht. Gesundheitswesen 2018. doi:10.1055/ a-0592-7184

[16] Seidling HM, Send AFJ, Bittmann J et al. Medication review in German community pharmacies - Post-hoc analysis of documented drug-related problems and subsequent interventions in the ATHINA-project. Research in Social and Administrative Pharmacy 2017; 13: 1127-1134. doi:10.1016/j.sapharm.2016.10.016 